\title{
A Comparative Study: Knowledge and Practices amongst Post-Operative Patients Regarding Hospital Acquired Infections (HAI) between Private and Public Tertiary Care Setup in Pakistan
}

\author{
Asna Shahab*, Tahir Sultan Shamsi ${ }^{2}$, Erum Afaq ${ }^{3}$, Omer Mustafa ${ }^{1}$, Daania Aman ${ }^{4}$ Omer Khan ${ }^{1}$, \\ Arisha Zaheerr, Safi Shaikh ${ }^{5}$, Rabiya $\mathrm{Irfan}^{4}$, Mustafa Saleem ${ }^{6}$, Shayan Marsiya ${ }^{6}$ \\ ${ }^{1}$ Department of Medicine, Dow University of Health Sciences, Karachi, Pakistan. \\ ${ }^{2}$ Department of Internal Medicine, National Institute of Blood Disease \& Bone Marrow Transplantation, Karachi, Pakistan.
}

${ }^{3}$ Department of Physiology, Liaquat National Hospital and Medical College, Karachi, Pakistan.

${ }^{4}$ Department of Surgery and Allied, Liaquat National Hospital and Medical College, Karachi, Pakistan.

${ }^{5}$ Department of Medicine, Liaquat National Hospital and Medical College, Karachi, Pakistan.

${ }^{6}$ Department of Surgery and Allied, Dow University of Health Sciences, Karachi, Pakistan.

\begin{abstract}
Background: Hospital Acquired Infection (HAI) is a major global safety concern for the health system as it increases mortality, morbidity, and length of hospital stay and contributes to the economic burden. These infections are prevalent in hospitals of developing countries such as Pakistan, due to limited resources, unsafe infection control practices and under reporting. However, post-operative patients are more prone to these infections due to immunocompromised state, antibiotic resistance, and most importantly lack of awareness regarding HAIs and their respective preventive measures. Therefore, the objective of this study was to highlight some of the serious but avoidable aspects of this largely ignored but important issue of HAIs in public and private tertiary care hospitals by assessing and comparing the need of knowledge and awareness in post-operative patients to minimize risks which in turn will decrease incidence, morbidity and mortality.
\end{abstract}

Materials and Methods: A descriptive cross sectional study was conducted among post-operative patients of Liaquat National Hospital and Civil Hospital (private and public tertiary care hospital, respectively), over a period of 1 year (July 2017-2018) in Karachi. Individual interviews following verbal consent were taken using a pre-coded questionnaire, which was divided into 3 sections to analyze the social and demographic, knowledge and practices of the patients. We excluded patients with impaired comprehension, less than 15 years of age and those who were reluctant to participate. Data was analyzed using SPSS v.22.0. Pearson's chi-square was used as the primary statistical test.

Results: Three-fifty post-operative patients from each, public and private hospital were enrolled. The mean age was found to be $38.41 \pm 16.57$ years and $40.3 \%$ of patients were uneducated. Knowledge regarding the modes of spread, types and risk factors for hospital acquired infections was significantly associated with patients admitted in private hospital, female gender and a higher education level $(\mathrm{P}<0.05)$.

Among the modes of spread of HAIs, dirty beds $(\mathrm{n}=666,95.1 \%)$ and dirty toilets $(\mathrm{n}=662,94.6 \%)$ were widely identified and respiratory tract infection (522 (74.9\%) was the most commonly identified type of HAIs. It was also noted that there were misconceptions and fears seen among $37.7 \%$ of patients regarding the prevention of HAIs.

Perceptions towards HAIs were generally sound with $96.3 \%$ respondents agreeing to report previous infections. While about 208 (30\%) respondents disagreed with limiting visiting hours in hospital can minimize the risk of HAIs overall. Moreover, with regard to knowledge score, all perception responses reached a statistical significance.

A total of $616(88.0 \%)$ patients stated that during their hospitalization they had received no information concerning nosocomial infections. It is indeed noteworthy that $432(61.7 \%)$ patients had received information regarding HAIs outside healthcare units, television (25.7\%) being the most popular choice.

Conclusion: The study revealed that there was an overall lack of awareness and knowledge regarding HAIs, especially in participants from public hospital.

Thus, there is a need to initiate educational campaigns that create community awareness and help improve perceptions of people regarding this issue. Strict monitoring of infection control practices and counseling of the patients upon admission or during the stay can also help reduce the burden.

Keywords: Hospital acquired infection, Post-operative, Knowledge, Prevalence, Tertiary care set-up, Public and private hospital.

*Address correspondence to this author at the Department of Medicine, Dow

University of Health Sciences, Karachi, Pakistan.

E-mail:asna_s93@yahoo.com 


\section{INTRODUCTION}

Infections acquired in health-care settings, are commonly referred to as Hospital Acquired Infections, they are amongst the most frequent adverse event in health-care delivery worldwide.

According to WHO's published health care-associated infections FACT SHEET, out of every 100 hospitalized patients at any given time, 7 in the developed and 10 in the developing countries will acquire at least one health care-associated infection. In addition to that the Center for Disease Control and Prevention illustrates that HAIs contributes to approximately 99,000 deaths each year $[1,2]$.

The prevalence of Hospital acquired infections in low- and middle-income countries (LMICs) is supported with limited data of low quality. As per WHO analysis HAIs are more recurrent in resource-limited settings than in developed countries as at any given time, the prevalence of infection varies between $5.7 \%$ and $19.1 \%$ in low- and middle-income countries respectively [3].

Amongst many prevailing factors socio-economic conditions are a major contributor as patients in low and middle income countries like Pakistan, are at a higher risk for acquiring such infections because of lack of basic resources and facilities such as infection control measures, overcrowding, lack of trained personnel in hospital and largely underreporting [3, 4-7]. Moreover, infections seen in post-operative patients such as surgical site infections account for about $24 \%$ of all hospital acquired infections. The problem is further aggravated in developing countries like Pakistan where resources are scarce and staff always short in supply $[8,9]$.

Hospital acquired infections continues to be a challenge and threat to the safe and effective functioning of health systems and has negative impacts on the quality of health services being delivered. In addition to that it also adds to the economical burden by prolonging hospital stay, increasing incidence of long-term disability and the likelihood of emerging resistance of microorganisms to antimicrobials; in short adding a massive financial burden for the health-care systems as well as for the patients and their families and leading to a surge in mortality $[7,10]$.

Based on the data available, it is clear that HAI is a global issue and deserves attention. At the present there has been little assessment done regarding patient's knowledge and experiences concerning hospital-acquired infections and in particular on methods for their prevention. One hopes that gaining a better understanding of the patient's perspective will bring compelling changes in the public health policy and infection-control procedures. Therefore, our study aims to concurrently document and compare the level of knowledge and perception of the most prone population; post-operative patents in two contrasting settings; public and private tertiary care setups respectively.

\section{MATERIALS AND METHODS}

\section{Study Design}

We conducted a descriptive cross sectional study from July 2017 to July 2018 in Karachi, Pakistan. Two tertiary care hospitals were chosen, first being a private setup; Liaquat National Hospital and the second being a government setup; Civil Hospital. The objective of our study was to assess the knowledge and practices regarding hospital acquired infections (HAIs) prevalent amongst post-operative patients admitted in the respective hospitals. The sample population was selected through random sampling.

\section{Data Collection Tools}

A pre-designed and structured questionnaire was developed and used for data collection. The questionnaire was originally in English and was also translated in the local language Urdu, as it is commonly understood by the general population and would prevent any misinterpretation due to language barrier. The questionnaire comprised of 26 questions in total, out of which 10 were aimed at gathering information regarding social and demographic aspect of the patients. The other 7 variables analyzed the patient's knowledge, whereas the other 9 variables analyzed patient's health care practices.

The interviews were conducted one-on-one, each lasting about 10-15 minutes, whereas about 80 to 90 questionnaires were filled by the patients themselves.

\section{Participants and Setting}

Initially the sample size comprised of 737 patients in total, out of which 700 agreed to participate and were equally divided in two groups comprising of 350 patients from each healthcare setup. The sample size was determined by open epi (Table 1).

We interviewed adult patients with intact decision making capacity that had undergone a surgical procedure and had been admitted for more than two days (48 hours) in surgical care in the respective tertiary care hospital. Participants with limited comprehension, less than 15 years of age, those who could not speak, write or understand English/Urdu to comprehend the questions and those that were reluctant were excluded. Any participation from the attendant was not counted for, hence minimizing the respondent bias.

\section{Ethical Considerations}

Ethical approval was obtained from Liaquat National Hospital and Medical College Ethical Review Board. A verbal consent was obtained from all participants prior to undergoing the survey. 
Table 1. Sample Size: X-Sectional, Cohort, \& Randomized Clinical Trials

\begin{tabular}{|c|c|c|c|}
\hline \multicolumn{3}{|l|}{ Two-sided Significance Level(1-alpha) } & 95 \\
\hline \multicolumn{3}{|l|}{ Percent of Unexposed with Outcome } & 5 \\
\hline \multicolumn{3}{|l|}{ Percent of Exposed with Outcome } & 11 \\
\hline \multicolumn{3}{|l|}{ Risk/Prevalence Ratio } & 2.2 \\
\hline \multicolumn{3}{|l|}{ Risk/Prevalence Difference } & 6.1 \\
\hline & Kelsey & Fleiss & Fleiss with $\mathrm{CC}$ \\
\hline Total Sample Size & 620 & 618 & 682 \\
\hline \multicolumn{4}{|c|}{$\begin{array}{l}\text { References } \\
\text { Kelsey et al., Methods in Observational Epidemiology 2nd Edition, Table 12-15. } \\
\text { Fleiss, Statistical Methods for Rates and Proportions, formulas } 3.18 \& 3.19 \text {. } \\
\text { CC = continuity correction. }\end{array}$} \\
\hline
\end{tabular}

\section{RESULTS}

Of the 737 patients approached to participate in the study, a total of 700 agreed to be interviewed with an overall response rate of $94.9 \%$. The distribution of relevant demographic characteristics of participants is summarized in Table 2.

Our study comprised of both private and public sector hospitals with fairly equal participation of males and females. The majority of the participants were adults within the age group of 18 to 64 years with the mean age being $38.41 \pm 16.57$ years. Of the 700 patients interviewed, 252(36.0\%) were admitted for the first time in the surgical ward while the remaining had been admitted either between one to five times (55.7\%), five to ten times $(6.60 \%)$ or more than 10 times $(1.20 \%)$. Moreover, only $54(7.7 \%)$ had a history of working for healthcare.

Overall, the mean score in the level of education was significantly higher for patients admitted in private hospitals as compared to the patients admitted in public hospitals

Table 2. Demographic Characteristics of the Study Population. (Note: $p$ values $<0.05$ is statistically significant).

\begin{tabular}{|c|c|c|c|c|c|}
\hline & Hospital Type & & Total n (\%) & Knowledge Score & p value \\
\hline & Public n (\%) & Private n (\%) & & & \\
\hline \multicolumn{6}{|l|}{ Gender } \\
\hline Male & $170(48.6 \%)$ & $158(45.1 \%)$ & $328(46.9 \%)$ & $13.06 \pm 3.90$ & 0.012 \\
\hline Female & $180(51.4 \%)$ & $192(54.9 \%)$ & $372(53.1 \%)$ & $13.81 \pm 3.44$ & \\
\hline \multicolumn{6}{|l|}{ Age groups } \\
\hline Young $(<18$ years $)$ & $22(6.3 \%)$ & $18(5.1 \%)$ & $40(5.70 \%)$ & $12.00 \pm 4.52$ & $>0.05$ \\
\hline Adults (18-64 years) & $306(87.4 \%)$ & $280(80.0 \%)$ & $586(83.7 \%)$ & $13.51 \pm 3.70$ & \\
\hline Old $(>65)$ & $22(6.3 \%)$ & $52(14.9 \%)$ & $74(10.6 \%)$ & $13.81 \pm 2.72$ & \\
\hline \multicolumn{6}{|l|}{ Ethnic background } \\
\hline Sindhi & $92(26.3 \%)$ & $44(12.6 \%)$ & $136(19.4 \%)$ & $12.56 \pm 4.17$ & 0 \\
\hline Punjabi & $30(8.6 \%)$ & $54(15.4 \%)$ & $84(12.0 \%)$ & $13.48 \pm 3.43$ & \\
\hline Muhajir & $98(28.0 \%)$ & $118(33.7 \%)$ & $216(30.9 \%)$ & $14.69 \pm 3.22$ & \\
\hline Pashto & $60(17.1 \%)$ & $78(22.3 \%)$ & $138(19.7 \%)$ & $12.55 \pm 3.70$ & \\
\hline Others & $70(20 \%)$ & $56(16.0 \%)$ & $126(18.0 \%)$ & $13.29 \pm 3.45$ & \\
\hline \multicolumn{6}{|l|}{ Education level } \\
\hline Uneducated & $186(53.1 \%)$ & $96(27.4 \%)$ & $282(40.3 \%)$ & $12.35 \pm 4.01$ & 0 \\
\hline Matriculation & $84(24.0 \%)$ & $86(24.6 \%)$ & $170(24.3 \%)$ & $13.76 \pm 3.29$ & \\
\hline Undergraduate & $34(9.8 \%)$ & $92(26.3 \%)$ & $126(18.0 \%)$ & $14.86 \pm 2.78$ & \\
\hline Postgraduate & $46(13.1 \%)$ & $76(21.7 \%)$ & $122(17.4 \%)$ & $14.13 \pm 3.48$ & \\
\hline
\end{tabular}


$(\mathrm{p}=0.001)$. Females $(\mathrm{x}: 13.81 \pm 3.44)$ were observed to have higher mean knowledge score $(\mathrm{p}=0.012)$ as compared to males ( $\mathrm{x}: 13.06 \pm 3.90)$. In addition, undergraduate students (x: $14.86 \pm 2.78)$ were found to have higher mean knowledge scores than postgraduate $(\mathrm{x}: 14.13 \pm 3.48)$ and matriculation students (x: $13.76 \pm 3.29)$. Overall, educated patients had a moderately higher mean score in knowledge as compared to uneducated patients (x: $12.35 \pm 4.01)$.

Table 3 summarizes the participants' responses to questions regarding assessment of knowledge about hospital acquired infections in relation to the type of hospital. Dirty beds $(\mathrm{n}=666,95.1 \%)$ and dirty toilets $(\mathrm{n}=662,94.6 \%)$ were among the most commonly identified potential modes of spread of HAIs. However, around one-third of the population falsely

Table 3. Responses to Questions Regarding Knowledge on Hospital Acquired Infections and their Correlation with Hospital Type. (Note: $p$ values $<0.05$ are statistically significant).

\begin{tabular}{|c|c|c|}
\hline Question & Yes n $(\%)$ & No n $(\%)$ \\
\hline \multicolumn{3}{|l|}{ What are the potential modes of spread of hospital acquired infections } \\
\hline Airborne & $542(77.4 \%)$ & $158(22.6 \%)$ \\
\hline Antibiotic overuse & $440(62.9 \%)$ & $260(37.1 \%)$ \\
\hline Dirty beds & $666(95.1 \%)$ & $34(4.90 \%)$ \\
\hline Skin contact & $574(82.0 \%)$ & $126(18.0 \%)$ \\
\hline Unsterilized equipment & $616(88.0 \%)$ & $84(12.0 \%)$ \\
\hline Dirty toilets & $662(94.6 \%)$ & $38(5.40 \%)$ \\
\hline Visitors & $476(68.0 \%)$ & $224(32.0 \%)$ \\
\hline Improper wound dressing & $600(85.7 \%)$ & $100(14.3 \%)$ \\
\hline \multicolumn{3}{|l|}{ Which of the following count as a hospital acquired infection } \\
\hline Urinary Tract Infection & $436(62.3 \%)$ & $264(37.7 \%)$ \\
\hline Surgical site infection & $446(63.7 \%)$ & $254(36.3 \%)$ \\
\hline Blood stream infection & $396(56.6 \%)$ & $304(43.4 \%)$ \\
\hline Respiratory tract infection & $522(74.9 \%)$ & $178(25.4 \%)$ \\
\hline Gastrointestinal infection & $444(63.4 \%)$ & $256(36.6 \%)$ \\
\hline Are hospital acquired infections preventable & $436(62.3 \%)$ & $264(37.7 \%)$ \\
\hline Having a chronic disease increases your risk of hospital acquired infection & $484(69.1 \%)$ & $216(30.8 \%)$ \\
\hline Prolonged stay at a hospital increases your risk of infection & $532(76.0 \%)$ & $168(24.0 \%)$ \\
\hline Compromised immune/nutritional status increases your risk of infection & $586(83.7 \%)$ & $114(16.30 \%)$ \\
\hline Being age $>65$ years increases your risk of infection & $562(80.3 \%)$ & $138(19.7 \%)$ \\
\hline
\end{tabular}

believed that antibiotic overuse (37.1\%) and visitors (32.0\%) could not contribute to their spread.

With the exception of dirty beds, skin contact, dirty toilets and visitors, all of the other modes of transmission were better correctly identified by participants from private hospital. When assessing knowledge based on the types of hospital acquired infections, the one most commonly identified was respiratory tract infection by around three quarters of the participants. Moreover, participants from private hospital had a better understanding of urinary ( $p$-value $=0.00)$ and respiratory tract infection ( $\mathrm{p}$-value $=0.000)$ as a type of HAI. Well over one-third $(37.7 \%)$ of the patients falsely believed that HAIs are not preventable. As for the risk factors such as having a chronic disease $(\mathrm{p}=0.000)$, prolonged hospital admission time ( $\mathrm{p}$-value $=0.001)$, compromised immune status $(p$-value $=0.000)$ and old age $(p$-value $=0.005)$, patients from private hospital were more likely to acknowledge them.

Perception of participants regarding HAIS is displayed in Fig (1). Among the respondents, the overwhelming majority agreed to the fact that it is necessary to report any history of previous infection to your healthcare worker. When stratified with hospital type, there was statistical difference observed for not sharing infected personal items such as towels and razors $(p$-value $=0)$, completion of antibiotic course post discharge ( $p$-value $=0.019)$, better staff compliance leading to reduced incidence of HAIs ( $\mathrm{p}$-value $=0.011$ ), restricting patients movements between wards (p-value $=0.001$ ) and limiting the visiting hours as a measure to reduce HAIs ( $p$-val- 
Making alcohol rubs for all visitors mandatory would decrease the incidence of hospital acquired infections

\section{Limiting visiting hours would minimize the risk of hospital acquired infections}

Restricting patients' movement from ward to ward will decrease the risk of hospital acquired infections

Better staff compliance with infection control measures (hand hygeine, aprons, sterile tools, etc) will decrease the risk of hospital acquired infections

Increased domestic cleaning staff decreases the risk of hospital acquired infections

Taking all of your antibiotics as directed after discharge and completing the dose is important

If infected, not sharing personal items like towels and razors is important

If infected previously, telling your health care providers is necessary

If left untreated, these infections can become life threatening


68

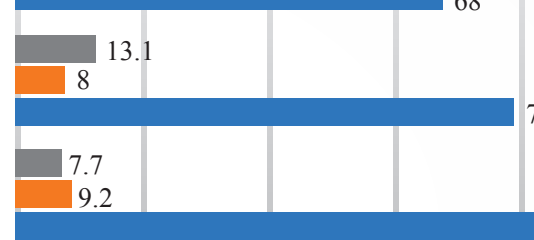

78.9
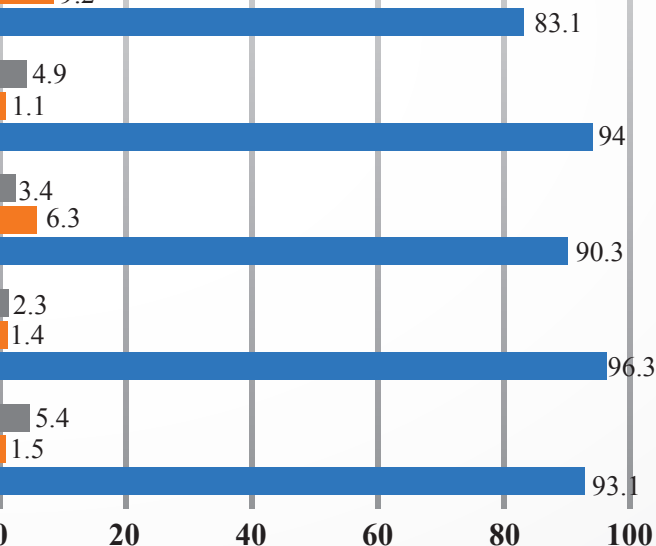

120

Uncertain Disagree Agree

Fig. (1). Responses to Questions Regarding Perceptions Towards Hospital Acquired Infections.

$\mathrm{ue}=0$ ). Similarly, when stratified with gender, there was statistical difference observed for not sharing personal items such as towels and razors ( $\mathrm{p}$-value $=0$ ) as more than half of female respondents $(50.3 \%)$ agreed. It was also seen that educated patients acknowledged it better than uneducated patients $(p$-value $=0.003)$ the importance of informing one's healthcare worker about any previous infection. With regard to knowledge score, all perception responses were statistically significant.

The graph above depicts that a considerable number of female respondents recognized urinary tract infection as the most common type of HAI in comparison to male respondents (Fig. 2).

On inquiring about the main sources of information, by which knowledge and awareness of hospital acquired infections was obtained, $432(61.7 \%)$ of the patients recorded that they had indeed learned about it outside hospital: Television was the most frequently mentioned mode of media (25.7\%) however, only $6.90 \%$ of the patients learned about it from local clinics.

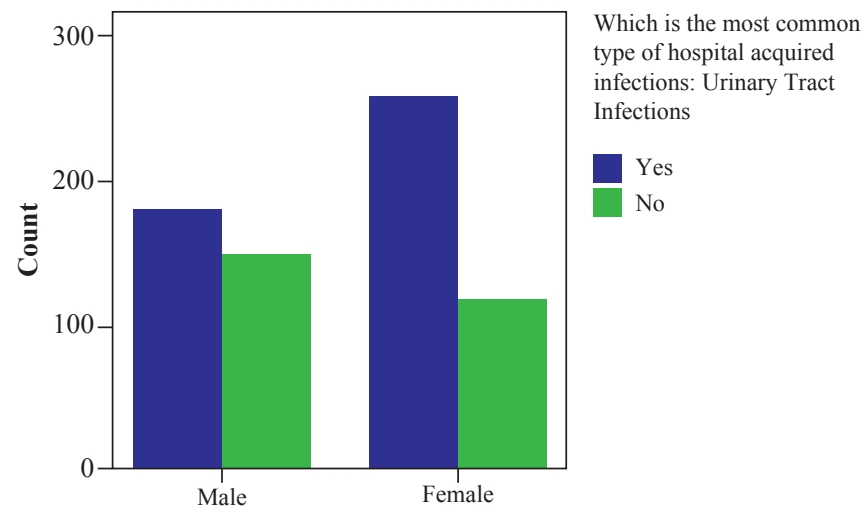

Fig. (2). Response to Urinary Tract Infection as the Most Common Type of HAI by Gender. 


\section{DISCUSSION}

The purpose of this study was to evaluate the differences in knowledge and perceptions regarding HAIs among postoperative patients in public and private tertiary care hospitals, respectively. The findings from this study suggested that, overall, patients admitted in the private healthcare setup were observed to be more up to date about the hospital-acquired infections and most recognized the well-known risk groups and risk factors for these infections. The study also found a significant association of patient's knowledge with their perception about prevention. Furthermore, more than half of the participants had learned about HAIs outside the hospitals; through mass media. Television was reported to be the main source of information.

Our study revealed that females presented with greater knowledge than males in regard to transmission, types and risk factors for such infections. There was a conflict with the findings of a study conducted by Abbate, et al. [11] in which they could not predict the gender with significant knowledge. Scoring differences between males and females could be due to the fact that more females in our society tend to stay at homes and therefore have a greater access to television. Association of age groups with knowledge was proposed by Mattner, et al. [12] however, our findings did not find age to be an important factor. Moreover, our study found a correlation between a high level of educational attainment as to having a better understanding of these infections, which coincides with surveys conducted by, Merle, et al. [13], Abbate, et al. [11] and Ocran, et al. [14]. This indicates that general awareness regarding hospital acquired infections is not yet largely prevalent.

A vast majority of patients displayed superior knowledge about the potential modes of transmission of HAIs. This is in contrary to a pilot study conducted in the United Kingdom $[15,16]$. These differences may be attributed to the participant's level of education and personal experience.

One of the key findings of our study was that the modes of transmission such as airborne, antibiotic overuse, unsterilized equipment and dirty toilets were better correctly identified by participants from private hospital. This is reinforced by the fact that preference of private health-care setups in Pakistan is linked to higher socioeconomic status and level of education of patients, respectively [17-19]. Therefore, these patients better understand how unnecessary usage of antibiotics lead to bacterial resistance and that a non-sterile equipment can be a source of infection. In Pakistan, private healthcare setups provide better services to their patients in terms of aseptic techniques, trained staff, laundry facilities, strict visiting hours and rules $[18,19]$. Therefore patients admitted were less likely to experience skin contact, dirty beds, improper dressing of wounds or visitors as possible modes of spread.
It would appear, that knowledge about the types of hospital acquired infection was moderate among the participants. For instance more than one-third post-operative patients were unaware of the possibility that they could acquire a surgical site infection. However, urinary and respiratory tract infections were better correctly identified by patients from private hospital. This result is not much to our surprise as urinary tract and respiratory tract infections are the most commonly reported type of hospital acquired infections [20,21].

Our findings also showed that female respondents recognized urinary tract infection more than their male counterparts. This is affirmed by the fact that UTIs are one of the most frequently occurring clinical infections in women, accounting for nearly $25 \%$ of all infections. Around $50-60 \%$ of women experiences a UTI in their lifetime, suggestive of their knowledge in this regard [22].

There were mixed opinions about whether HAIs are preventable. More than one-third of the participants were under false impression that they are not. This differed from Abbate, et al. findings whereby only $88 \%$ of the participants believed that they are preventable [11]. This indicates patient's apprehensions about the severity of the disease. Such participants displayed inferior knowledge about the modes of spread, types and risk factors, as opposed to those who perceived otherwise.

Most patients recognized the well-known risk groups and risk factors for these infections, although it was also quite noteworthy that there are some misconceptions regarding them. Among risk groups, elderly were widely identified. This was consistent with the results reported by Abbate, et al. [11].

Positive correlation between rates of hospital acquired infections and average length of stay have been reported in various studies $[23,24]$. Likewise more than three quarter of participants in our survey viewed it as a risk factor. Interestingly, this disagreed with Abbate, et al. who found that more than half of participants interviewed did not recognize length of hospital stay as a likely cause of HAIs. This could be due to the fact that our survey was conducted on postoperative patients who tend to have prolonged hospital stays. Therefore it is possible that they estimated it as a risk, since they are selected among those who have no choice to avoid health care services.

Our study also evaluated the influence of knowledge score on various perception regarding hospital acquired infections. It was evident from the survey that those patients who had a better understanding of the subject were more likely to perceive and practice precautionary behaviors. From a list of nine perceptions, a striking majority was seen to appreciate the necessity of reporting infections to their healthcare worker. Similarly, a good majority believed that improving 
hospital hygiene and compliance with infection control measures would minimize the risks of HAIs. This is comparable to studies conducted in Europe where healthcare infrastructure is already very advanced $[11,15,16]$ Contrary to a study discussing the importance of limiting visiting hours in prevention of HAIs [16], our findings did not strongly reflect this proposition; whereby about one-third did not feel the need for its implementation. This is not surprising as Pakistani's are known to be very family oriented and restricting their family/friends could result in feelings of isolation and abandonment. Moreover, notion about restricting a patient's a movement from one ward to another was seen with some uncertainty. This was reported mostly by patients in public hospital, where it is a common practice for patients to move around and socialize.

These observations could possibly point towards lack of education and awareness which give rise to such misconceptions. This suggests that if patients are explained about the severity and the potential complications of these infections, their opinions can be changed. This can be achieved by carrying out mass awareness campaigns and information sessions with the patients.

Indeed, it is eminent that the main source of information to participants, through which awareness was attained was the media and to a lesser extent by qualified health care representatives. This is probably related to the high impact of exposure these sources have. The same is observed in surveys [11, 16, $25,26]$ whereby patients learned about HAIs from various media sources. Furthermore, the high proportion of patients who stated that they had received no information about the risks of HAIs upon admission is also noted in a survey published in 1989 [27] and a study conducted in France where information about infection risks is mandatory prior to surgery [13]. Moreover, no correlation of work experience in a healthcare setting with knowledge regarding HAI was found. This was in contrast with the survey [12] where authors noted an association with the healthcare setting was a common mode of information.

\section{LIMITATIONS}

Our study had some limitations within which our findings need to be interpreted carefully. First our sample size was restricted to only one public and one private tertiary healthcare setup respectively, from a large metropolitan city. Consequently, the results cannot predict levels of awareness and perceptions from other types of healthcare setups present in the city. Similarly, these results might not apply to patients residing in rural areas where lack of education, different cultural beliefs and a scarcity of healthcare facilities may have sturdy influences. Secondly, our participants consisted of patients who were admitted in surgical ward only and so the findings were not applicable to patients admitted in other wards of the hospital. Many patients seemed to be in discom- fort after the procedure, thus the bias and hesitations might have affected the conclusions. Furthermore, the research material available was not sufficient enough hence various aspects of the subject could not be covered. Therefore, we urge further study designs to be carried out countrywide. Nevertheless, these findings are crucial in assessing the comparison of knowledge and perception concerning hospital-acquired infections of post-operative patients in both public and private tertiary care hospitals.

\section{CONCLUSION}

In conclusion, current level of knowledge and perception of patients were seen to be above average in general, but the sources of information available regarding this matter is limited. In addition, the large knowledge gap between the two sectors was prominently evident. This calls for a national approach and managerial support from healthcare authorities to initiate awareness campaigns in public hospitals and a wider platform, that should include more accessible modes of communication that are easier to comprehend for individual's from different educational background and encourage making it mandatory for healthcare-workers to hold multiple counseling sessions with their patients upon admission and during the stay.

\section{CONFLICT OF INTEREST}

Declared none.

\section{ACKNOWLEDGEMENTS}

Declared none.

\section{AUTHORS' CONTRIBUTION}

All authors have contributed equally.

\section{REFERENCES}

[1] Klevens RM, Edwards JR, Richards CL, et al. Estimating Healthcare-associated Infections and Deaths in U.S. Hospitals, 2002. Public Health Rep 2007; 122(2): 160-6.

DOI: $10.1177 / 003335490712200205$

[2] Sueters C, Latour K, Karki T, et al. Prevalence of healthcare-associated infections, estimated incidence and composite antimicrobial resistance index in acute care hospitals and long-term care facilities: results from two European point prevalence surveys, 2016 to 2017. Euro Surveill 2018; 23: 46. DOI: 10.2807/1560-7917.ES.2018.23.46.1800516

[3] WHO. Health care-associated infections fact sheet. Available at: https://www.who.int/gpsc/country_work/gpsc_ccisc_fact_sheet_en.pdf [20 April 2020].

[4] Nejad SB, Allegranzi B, Syed SB, Ellis B, Pittet D. Health-care-associated infection in Africa: A systemic review. Bull World Health Org 2011; 89: 757-65.

DOI: $10.2471 /$ BLT.11.088179 
[5] Ali S, Birhane M, Bekele S, et al. Healthcare associated infection and its risk factors among patients admitted to a tertiary hospital in Ethiopia: Longitudinal study. Antimicrob Resist Infect Control 2018; 7: 2.

DOI: $10.1186 / \mathrm{s} 13756-017-0298-5$

[6] Damani N. Simple measures save lives: An approach to infection control in countries with limited resources. J Hospital Infect 2007; 65: 151-4.

DOI: 10.1016/S0195-6701(07)60034-6

[7] Sastry S, Masroor N, Bearman G, et al. The $17^{\text {th }}$ International Congress on Infectious Diseases workshop on developing infection prevention and control resources for low- and middle-income countries. Int J Infect Dis 2017; 57: 138-43. DOI: $10.1016 /$ j.ijid.2017.01.040

[8] Agarwal M, Thomas P. Prevalence of post-op. nosocomial infection in neuro-surgical patients and associated risk factors - A prospective study of 2441 patients. Nurs J India 2003; 94 : 197-8.

[9] Ahmed MI. Prevalence of nosocomial wound infection among postoperative patients and antibiotics patterns at teaching hospital in Sudan. N Am J Med Sci 2012; 4(1): 29-34.

DOI: $10.4103 / 1947-2714.92900$

[10] WHO Programs. Clean Care is Safer Care [internet]. [Cited Oct 9, 2017]. Available from: http://www.who.int/gpsc/country_work/burden_hcai/en/ [Accessed October 9, 2017].

[11] Abbate G, DiGiuseppe O, Marinelli I, Angelillo IF, Collaborative working group. Patient's knowledge, attitudes, and behavior toward hospital-associated infections in Italy. Am J Infect Control 2008; 36: 39-47. DOI: 10.1016/j.ajic.2007.01.006

[12] Mattner F, Mattner C, Zhag I, Gastmeier P. Knowledge of nosocomial infections and multiresistant bacteria in the general population: Results of a street interview. J Hosp Infect 2006; 62: 524-5. DOI: 10.1016/j.jhin.2005.09.027

[13] Merle V, Rossem VV, Tavolacci MP, Czernichow P. Knowledge and opinions of surgical patients regarding nosocomial infections. J Hosp Infect 2005; 60: 169-71.

DOI: $10.1016 /$ j.jhin.2004.09.003

[14] Ocran I, Tagoe DNA. Knowledge and attitude of healthcare workers and patients on healthcare associated infections in a regional hospital in Ghana. Asian Pac J Trop Dis 2014; 4(2): 135-9. DOI: 10.1016/S2222-1808(14)60330-3

[15] Minahan J B. Ethnic Groups of South Asia and the Pacific: An Encyclopedia. ABC-CLIO: Santa Barbara 2012.

[16] Madeo M, Shields L, Owen E. A pilot study to investigate patient's reported knowledge, awareness and beliefs about health care-associated infection. Am J Infect Control 2008; 36 : 63-9. DOI: 10.1016/j.ajic.2007.01.008

[17] Naseer M, Zahidie A, Shaikh BT. Determinants of patient's satisfaction with health care system in Pakistan: A critical review. Pak J Public Health 2012; 2(2): 52-61.

[18] Hussain A, Sial MS, Usman SM, Hwang J, Jiang Y, Shafiq A. What factors affect patient satisfaction in public sector hospitals: Evidence from an emerging economy. Int J Environ Res Public Health 2019; 16(6): 994.

DOI: $10.3390 /$ ijerph16060994

[19] Irfan SM, Ijaz A. comparison of service quality between private and public hospitals: Empirical evidences from Pakistan. J Qual Technol Manag 2011; 7: 1-22.

[20] Ducel G, Fabry J, Nicolle L. Prevention of hospital-acquired infections, a practical guide. $2^{\text {nd }}$ ed. Geneva: WHO/CDS/CSR/EPH 2002.

[21] Foxman B, Barlow R, D'Arcy H, Gillespie B, Sobel JD. Urinary tract infection: Self-reported incidence and associated costs. Ann Epidemiol 2000; 10(8): 509-15.

DOI: $10.1016 / \mathrm{S} 1047-2797(00) 00072-7$

[22] Esen S, Leblebicioglu H. Prevalence of nosocomial infections at intensive care units in Turkey: A multicentre 1-day point prevalence study. Scand J Infect Dis 2004; 36: 144-8.

DOI: $10.1080 / 00365540410019156$

[23] Craven DE, Kunches LM, Lichtenberg DA, et al. Nosocomial infection and fatality in medical and surgical intensive care unit patients. Arch Intern Med 1988; 148: 1161-8.

DOI: $10.1001 /$ archinte.148.5.1161

[24] Dasgupta S, Das S, Chawan NS, Hazra A. Nosocomial infections in the intensive care unit: Incidence, risk factors, outcome and associated pathogens in a public tertiary teaching hospital of Eastern India. Indian J Crit Care Med 2015; 19(1): 14-20. DOI: $10.4103 / 0972-5229.148633$

[25] Gammon J. Analysis of the stressful effects of hospitalization and source isolation on coping and psychological constructs. Int J Nurs Pract 1998; 4: 84-96.

DOI: $10.1046 /$ j.1440-172X.1998.00084.x

[26] Miller PJ, Farr BM. Survey of patient's knowledge of nosocomial infections. Am J Infect Control 1989; 17: 31-4. DOI: $10.1016 / \mathrm{S} 0196-6553(89) 80010-0$

[27] Bouza E, Alousa S, Asensio A, et al. Information on nosocomial infections in the mainstream media: An opinion document. Rev Esp Quimioter 2019; 32(2): 165-77. 